\title{
A Note on Transitive Permutation Groups of Degree Twice a Prime
}

\author{
Primož Potočnik \\ Faculty of Mathematics and Physics, University of Ljubljana, and \\ Institute of Mathematics, Physics, and Mechanics \\ Jadranska 19, 1000 Ljubljana, Slovenia \\ Mateja Šajna* \\ Department of Mathematics and Statistics, University of Ottawa \\ 585 King Edward Avenue, Ottawa, ON, K1N 6N5, Canada
}

Received 3 August 2008, accepted 4 November 2008, published online 8 December 2008

\begin{abstract}
In this note, we consider transitive permutation groups of degree $2 p$, where $p$ is an odd prime, that admit blocks of imprimitivity of size 2 but no blocks of imprimitivity of size $p$.
\end{abstract}

Keywords: Permutation group, imprimitive group.

Math. Subj. Class.: $20 B 05$

Permutation groups of some special degrees have been a subject of investigation of several distinguished authors, including Burnside who classified permutation groups of prime degree (see for example [1, Corollary 3.5B]). A natural next step, a classification of primitive permutation groups of twice a prime degree, was first attempted by Wielandt [9], and completed as a consequence of the classification of finite simple groups.

Recently several authors have become interested in the study of imprimitive permutation groups of some simple composite degrees. For example, permutation groups of prime-power degree were investigated in [3, 4], while imprimitive permutation groups of degree twice a prime were studied by Lefèvre [5], Marušič [6], and Marušič and Potočnik [7].

In this note we present a new result on imprimitive permutation groups of degree twice a prime that serves as a crucial tool in the classification of homogeneously almost selfcomplementary graphs of order four times a prime in [8]. We use standard terminology and notation as introduced in [9] and [8].

\footnotetext{
*This author gratefully acknowledges support by the Natural Sciences and Engineering Research Council of Canada.

E-mail addresses: primoz.potocnik@fmf.uni-lj.si (Primož Potočnik), msajna@uottawa.ca (Mateja Šajna)
} 
Theorem 1. Let $p$ be an odd prime, $V$ a set of size $2 p, G$ a transitive permutation group on $V$, and $P$ a Sylow p-subgroup of $G$. Then $P$ has two orbits on $V$, each of size $p$. Suppose further that the orbits of $P$ are not blocks of imprimitivity for $G$, but that there exists a $G$ invariant partition $\mathcal{B}$ of $V$ into blocks of size 2 . Let $K=\operatorname{Ker}\left(G \rightarrow G^{\mathcal{B}}\right)$ denote the kernel of the induced action of $G$ on $\mathcal{B}$. Then one of the following occurs:

(i) $|K| \leq 2, G^{\mathcal{B}}$ is a non-solvable 2-transitive group, and for every $B \in \mathcal{B}$ and $v \in B$ the stabilizer $G_{v}$ acts transitively on the set $V \backslash B$;

(ii) $|K| \geq 4$ and either

(a) $p=3,|K|=4$; or

(b) for any $B, B^{\prime} \in \mathcal{B}$ there exist a third block $B^{\prime \prime} \in \mathcal{B} \backslash\left\{B, B^{\prime}\right\}$ and a permutation $\tau$ in $K$ acting non-trivially on each of $B, B^{\prime}$, and $B^{\prime \prime}$.

In both cases, for any two blocks $B, B^{\prime} \in \mathcal{B}$ there exists a permutation in $G$ fixing $B$ pointwise and $B^{\prime}$ setwise but not pointwise. Moreover, in Case (ii) such a permutation exists in $K$.

Proof. Since the size of every $P$-orbit is a power of $p$ and the size of a smallest $P$-orbit is $p$ by [9, Theorem 3.4], the group $P$ has indeed exactly two orbits on $V$, each of size $p$. Now, let $\mathcal{B}$ be a $G$-invariant partition of $V$ into blocks of size 2 and $f: G \rightarrow G^{\mathcal{B}}$ the natural group epimorphism with kernel $K$. Since the order of $K$ is a power of 2, the intersection $K \cap P$ is trivial and $P \cong f(P) \cong P^{\mathcal{B}}$. In particular, $P$ is isomorphic to a $p$-subgroup of the symmetric group $\operatorname{Sym}(p)$ and is therefore of order $p$. Choose a generator $\rho$ of $P$ and a point $v \in V$. Let $v^{\prime}$ denote the unique point in $V$ such that $B=\left\{v, v^{\prime}\right\} \in \mathcal{B}$, and for all $i \in \mathbb{Z}_{p}$, let $v_{i}=v^{\rho^{i}}$, $v_{i}^{\prime}=v^{\prime \rho^{i}}$, and $B_{i}=\left\{v_{i}, v_{i}^{\prime}\right\}$. Then $\mathcal{B}=\left\{B_{i} \mid i \in \mathbb{Z}_{p}\right\}$. We shall first prove the following statement:

(A): If $G^{\mathcal{B}}$ is non-solvable, then it is 2-transitive, and for every $B \in \mathcal{B}$ and $u \in B$ the stabilizer $G_{u}$ acts transitively on the set $V \backslash B$. In particular, for any $B, B^{\prime} \in \mathcal{B}$ there exists a permutation in $G$ fixing $B$ pointwise and $B^{\prime}$ setwise but not pointwise.

Assume therefore that $G^{\mathcal{B}}$ is non-solvable. Then it is 2-transitive by a well-known theorem of Burnside (see for example [1, Corollary 3.5 B]). Clearly, the set $\overrightarrow{\mathcal{B}}=\left\{\left(w, w^{\prime}\right) \mid\right.$ $\left.\left\{w, w^{\prime}\right\} \in \mathcal{B}\right\}$ is a self-paired $G$-orbital on $V$ (that is, an orbit of the natural action of $G$ on $V^{[2]}$ with $\left(w, w^{\prime}\right) \in \overrightarrow{\mathcal{B}}$ if and only if $\left.\left(w^{\prime}, w\right) \in \overrightarrow{\mathcal{B}}\right)$. Let us prove that there exists at least one more self-paired $G$-orbital on $V$.

Suppose, on the contrary, that all other $G$-orbitals are non-self-paired. This, in particular, implies that every orbit of size 2 of every permutation in $G$ has to be of the form $\left\{w, w^{\prime}\right\} \in \mathcal{B}$. Since $G^{\mathcal{B}}$ is 2-transitive, there exists $\alpha \in G$ swapping two blocks $B_{i}, B_{j} \in \mathcal{B}$. Moreover, by taking an appropriate odd power of $\alpha$, we may assume that the order of $\alpha$ is a power of 2. Since $|\mathcal{B}|$ is odd, $\alpha$ fixes setwise at least one block $B^{\prime} \in \mathcal{B}$. Since $\alpha$ has no orbits of size 2 on $B_{i} \cup B_{j}$, its square $\alpha^{2}$ fixes each of $B_{i}$ and $B_{j}$ setwise but neither of them pointwise, while fixing $B^{\prime}$ pointwise. Since $G^{\mathcal{B}}$ is 2 -transitive, there exists an element $\beta \in G$ swapping $B^{\prime}$ and $B_{j}$. However, observe that either $\beta$ or $\alpha^{2} \beta$ has two orbits of size 2 between $B^{\prime}$ and $B_{j}$, contradicting the assumption that there are no self-paired $G$-orbitals on $V$ other than $\overrightarrow{\mathcal{B}}$.

Now, let $\Delta$ be a self-paired $G$-orbital distinct from $\vec{B}$, and let $X=\operatorname{DiGr}(V, \Delta)$ be the corresponding orbital digraph of $G$. Since $\Delta$ is self-paired, $X$ is in fact a graph. To show that $G_{u}$ acts transitively on $V \backslash B$ for any $B \in \mathcal{B}$ and $u \in B$, it suffices to prove that $\Delta=\{(u, v) \mid u, v \in V, u \neq v\} \backslash \overrightarrow{\mathcal{B}}$, or equivalently, that $X$ is isomorphic to the lexicographic 


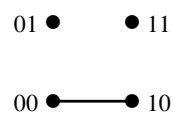

$\mathrm{L}$

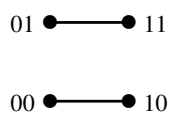

M

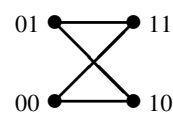

$\mathrm{C}$

Figure 1: Graphs L, M, and C.

product $K_{p}\left\langle K_{2}{ }^{\mathrm{c}}\right.$. To this end, consider the sets $\Delta_{i, j}$ of ordered pairs $(u, v) \in \Delta$ such that $u, v \in B_{i} \cup B_{j}, i, j \in \mathbb{Z}_{p}, i \neq j$, and the corresponding graphs $X_{i, j}=\operatorname{DiGr}\left(B_{i} \cup\right.$ $\left.B_{j}, \Delta_{i, j}\right)$. Since $G^{\mathcal{B}}$ is 2 -transitive, all these graphs are isomorphic to the same bipartite graph $\Gamma$. Moreover, since $G$ acts transitively on the $\operatorname{arcs}$ of $X$, the graph $\Gamma$ is arc-transitive and thus isomorphic to one of the graphs $\mathrm{L}, \mathrm{M}$, and $\mathrm{C}$ (see Figure 1). Let $\tau$ be the permutation on $V$ preserving each block in $\mathcal{B}$ setwise but not pointwise. This permutation clearly commutes with every permutation on $V$ preserving the partition $\mathcal{B}$. In particular, it centralizes the group $G$, and therefore $\Delta^{\tau}$ is also a $G$-orbital (note that $\Delta^{\tau}=\Delta$ if and only if $\Gamma \in\{\mathrm{M}, \mathrm{C}\}$ ). Let $\Delta^{\prime}=\Delta \cup \Delta^{\tau}$ and $Y=\operatorname{DiGr}\left(V, \Delta^{\prime}\right)$. Then $G \leq \operatorname{Aut}(Y)$. If the graph $\Gamma \in\{\mathrm{M}, \mathrm{L}\}$, then $Y$ is clearly a double cover over the complete graph $K_{p}$. However, by [6, Lemma 6.1], the orbits of $P$ on $V$ form a system of blocks of imprimitivity for $\operatorname{Aut}(Y)$, and therefore also for $G$, contradicting our assumptions. This shows that $\Gamma=\mathrm{C}$ and thus $X \cong K_{p} \prec K_{2}{ }^{\mathrm{c}}$ as required. Hence for any $B \in \mathcal{B}$ and $u \in B$, we have that $G_{u}$ acts transitively on $V \backslash B$. In addition, observe that for any two blocks $B, B^{\prime} \in \mathcal{B}$, the stabilizer $G_{v}$ of a vertex $v$ in $B$ contains a permutation mapping a vertex in $B^{\prime}$ to the other vertex in $B^{\prime}$. Such a permutation clearly fixes $B$ pointwise and $B^{\prime}$ setwise but not pointwise. The proof of Claim (A) is thus completed.

We would like to mention here that claim (A) can also be proved by using some rather different techniques, contained in [2, Theorem 2.4]; we thank the anonymous referee for pointing this out.

Now consider the kernel $K=\operatorname{Ker}\left(G \rightarrow G^{\mathcal{B}}\right)$. Suppose first that $|K| \leq 2$. If $G^{\mathcal{B}}$ is solvable, then its Sylow $p$-subgroup $P^{\mathcal{B}}$ is normal in $G^{\mathcal{B}}$ by [1, Corollary 3.5B], and so its preimage $f^{-1}\left(P^{\mathcal{B}}\right)=\langle P, K\rangle$ is normal in $G$. Since $K$ is normal in $G$ and hence normalized by $P$, which is of order $p$, the group $\langle P, K\rangle$ is cyclic of order $p$ or $2 p$. In either case, $P$ is characteristic in $\langle P, K\rangle$ and therefore normal in $G$. Consequently, the $P$-orbits on $V$ form a $G$-invariant partition of $V$, contradicting the assumption of the theorem. Hence $G^{\mathcal{B}}$ is nonsolvable and Claim (i) follows directly from (A).

We may therefore assume that $|K|>2$. Since $K$ is an elementary abelian 2-group, we have that $|K| \geq 4$. Let us first prove that for any two blocks in $\mathcal{B}$ there exists an element in $K$ acting trivially on any one of them and non-trivially on the other. If this is not the case, then there exist two blocks $B, B^{\prime} \in \mathcal{B}$ such that every element of $K$ fixing $B$ pointwise also fixes $B^{\prime}$ pointwise. Hence the pointwise stabilizers $K_{(B)}$ and $K_{\left(B^{\prime}\right)}$ are the same. Let $s \in \mathbb{Z}_{p}^{*}$ be such that $B^{\prime}=B^{\rho^{s}}$. Then $K_{(B)}=K_{\left(B^{\prime}\right)}=K_{(B)} \rho^{s}$, and since $\rho^{s}$ generates $P$, we can see that $K_{(B)}$ is normalized by $P$. Since, on the other hand, $P$ acts transitively by conjugation on $\left\{K_{\left(B^{\prime \prime}\right)} \mid B^{\prime \prime} \in \mathcal{B}\right\}$, it follows that $K_{\left(B^{\prime \prime}\right)}=K_{(B)}$ for every $B^{\prime \prime} \in \mathcal{B}$, and hence $|K|=2$, which contradicts our assumption. Therefore, for any two blocks in $\mathcal{B}$ there exists a permutation in $K$ fixing the first block pointwise and the second setwise but not pointwise. 
Now, if $p=3$, then either $|K|=4$ and we have Claim (a), or $|K|=8$ and we have Claim (b). We may therefore assume that $p \geq 5$. Let $B, B^{\prime} \in \mathcal{B}$ be arbitrary two blocks. As we have seen in the previous paragraph, there exist $\varphi, \psi \in K$ such that $\left.\varphi\right|_{B}$ and $\left.\psi\right|_{B^{\prime}}$ are trivial permutations while $\left.\varphi\right|_{B^{\prime}}$ and $\left.\psi\right|_{B}$ are non-trivial. The product $\vartheta=\varphi \psi$ then acts non-trivially on both $B$ and $B^{\prime}$. If $\vartheta$ acts non-trivially on any other block $B^{\prime \prime} \in \mathcal{B} \backslash\left\{B, B^{\prime}\right\}$, then the statement of the lemma holds. So we may assume that $\vartheta$ is trivial on all blocks in $\mathcal{B} \backslash\left\{B, B^{\prime}\right\}$. Let $\tau=\vartheta \vartheta^{\rho^{2}}$ if $B^{\prime}=B^{\rho}$, let $\tau=\vartheta \vartheta^{\rho^{-2}}$ if $B^{\prime}=B^{\rho^{-1}}$, and let $\tau=\vartheta \vartheta^{\rho}$ otherwise. It is now easy to see that $\tau$ acts non-trivially on $B, B^{\prime}$, and at least two other blocks in $\mathcal{B}$. This completes the proof of Claim (ii).

\section{References}

[1] J. D. Dixon and B. Mortimer, Permutation Groups, Graduate Texts in Mathematics 163, Springer, New York, 1996.

[2] E. Dobson, Automorphism groups of metacirculant graphs of order a product of two distinct primes, Combin. Prob. Comput. 15 (2006), 105-130.

[3] E. Dobson, On groups of odd prime-power degree that contain a full cycle, Discrete Math. 299 (2005), 65-78.

[4] E. Dobson and D. Witte, Transitive permutation groups of prime-squared degree, J. Algebraic Combin. 16 (2002), 43-69.

[5] C. Lefèvre, Une application de la théorie des codes aux groupes de rang 3 imprimitifs dont les blocs sont de longeur 2, Bull. Soc. Math. Belg. 26 (1974), 48-80.

[6] D. Marušič, On vertex symmetric digraphs, Discrete Math. 36 (1981), 69-81.

[7] D. Marušič and P. Potočnik, Semisymmetry of generalized Folkman graphs, Europ. Combin. 22 (2001), 333-349.

[8] P. Potočnik and M. Šajna, Brick assignments and homogeneously almost self-complementary graphs, J. Combin. Theory Ser B, to appear.

[9] H. Wielandt, Finite Permutation Groups, Academic Press, New York, 1964. 